\title{
Fresh Products Supply Chain Coordination Model under the Background of E-commerce Considering Freshness Affecting Demand
}

\author{
Zhiguo Fan, Yanjun Fan ${ }^{\mathrm{a}}$ \\ School of Management, Tianjin University of Technology, Tianjin, 300384, China \\ a E-mail:1208839223@qq.com
}

Keywords: e-commerce fresh products; supply chain coordination; freshness; quantity discount contract

\begin{abstract}
Considering fresh products with the characteristics of perishable and prone, this paper set up a demand function based on the freshness affecting demand. Firstly, the centralized decision model and the decentralized decision model are established by using the demand function, and the optimal price and profit under the two different decision models are analyzed. It is found that there is a profit margin in the e-commerce fresh products supply chain. On this basis, the coordination model of quantity discount contract is constructed. The research shows that the model can not only maximize the profit of supplier and e-commerce fresh products platform, but also coordinate the e-commerce fresh products supply chain. Finally, a numerical example was show to verify that the freshness of fresh products has direct influence on the profit of supply chain members and the validity and rationality of coordination model.
\end{abstract}

\section{Introduction}

With the rapid development of e-commerce and the improvement of people's consumption level, more and more people prefer purchasing online, which has promote the rapid development of e-commerce fresh products. According to Analysys International data, the total transaction volume of China's fresh e-commerce market in 2017 reached 139.13 billion yuan[1]. However, due to the fresh products with characteristics of perishable and the pursuit of high quality products by consumers, freshness has become the focus of consumers' attention in purchasing. Therefore, the construction of e-commerce fresh products supply chain coordination model considering freshness effect on demand is a new problem combining practice with theory.

At present, some scholars have done a lot of researches. In the aspect of double channel supply chain coordination, Dan Bin, et al [2] designed a reasonable coordination compensation strategy to realize the coordination of the dual-channel supply chain, which combines the electronic sales channel with the traditional retail channel. Amini and Li [3] studied the configuration of new product supply chain with two channels. In the aspect of agricultural products supply chain coordination, Dan Bin, Xu Guangye, et al [4] constructed the conditional risk value model with risk aversion characteristics to analyze the influence of adverse weather and the risk aversion of producers on the optimal input level of agricultural resources and coordination of supply chain. Tang run[5]studied on supply chain coordination of agricultural products under the influence of optimal preservation temperature adopted by suppliers on market demand. The above literature mainly focus on the analysis of manufacturing industry. When it comes to fresh products, it does not consider the influence of freshness on market demand, nor does it deal with the coordination of fresh products supply chain under the background of e-commerce. In view of this, considering the perishable characteristic of fresh products, this paper constructs a quantity discount contract decision model, which will provide constructive advice for supply chain members.

\section{Problem description and hypothesis}

In this paper, the e-commerce fresh products supply chain consisting of a single supplier and a 
single e-commerce fresh products platform is taken as the research object. Supplier provide or produce fresh products and sell them on e-commerce platform. Fresh products with the characteristics of perishable and prone will be affected by many factors in the process of picking, transportation, warehousing and sales. For convenience, it is assumed that market demand is affected only by freshness and price. Therefore, the market demand for fresh products on the e-commerce platform is expressed as follows [6]:

$$
d=a-f(\alpha) b p
$$

In the demand function, $a$ represents the basic demand of the fresh products market, $\mathrm{b}$ represents the elasticity index of the market demand to the price, and $p$ represents the sales price of the unit fresh products, $f(\alpha)=1 / \alpha, \alpha$ represents the freshness of fresh products, when $\alpha \rightarrow 1$, freshness has little effect on demand, when $\alpha \rightarrow 0$,freshness has a great impact on demand.

To simplify the problem, this paper makes the following hypothesis about model:

(1)The information between the supplier and the e-commerce fresh products platform is completely shared, both of them are rational and aim to maximize the profit.

(2) There is no shortage of fresh products, and distribution is carried out by supplier.

(3)The sales volume of fresh e-commerce is the market demand, and all orders are provided by supplier.

(4) This article only considers the freshness of fresh products sold on the e-commerce platform.

In addition, the associated parameter symbols are defined as:

The unit cost of fresh product is $c$; the wholesale price of unit fresh product is $w$; the profit of member of the supply chain is $\pi_{i}, i=t, s, e: t$ represents the supply chain system, s represents the supplier, e represents the e-commerce fresh products platform; the maximum fixed wholesale price is $K$; subscript "1" indicates the centralized decision model, subscript "2" indicates the decentralized decision model, subscript "3" indicates the coordination model.

\section{Decision Model of e-commerce fresh products supply chain considering freshness affecting demand}

\subsection{Centralized decision model}

Under the centralized decision model, the total profit of e-commerce fresh products supply chain system is obtained:

$$
\pi_{1 t}=(p-c) d_{1}=(p-c)(a-f(\alpha) b p)
$$

It can be seen from equation (2) that $\pi_{1 t}$ is binary concave function of $p$. Therefore, by finding the first derivative of function, we can obtain the optimal sales price as follows:

$$
p_{1}^{*}=\frac{a+f(\alpha) b c}{2 f(\alpha) b}, \pi_{1 t}^{*}=\frac{(a-f(\alpha) b c)^{2}}{4 f(\alpha) b}
$$

\subsection{Decentralized decision model}

Under the decentralized decision, members of the supply chain pursue their own profits maximization, so the profits of e-commerce fresh products platform and supplier are as follows:

$$
\begin{aligned}
& \pi_{2 e}=(p-w) d_{1}=(p-w)(a-f(\alpha) b p) \\
& \pi_{2 s}=(w-c) d_{2}=(w-c)(a-f(\alpha) b p)
\end{aligned}
$$

According to the principle of master-slave game to solve the above model, we can get the optimal selling price, the optimal demand and the optimal wholesale price of the e-commerce 
platform under the decentralized decision:

$$
p_{2}{ }^{*}=\frac{a+f(\alpha) b w}{2 f(\alpha) b}, \quad d_{2}^{*}=\frac{a-f(\alpha) b w}{2}, \quad w_{2}^{*}=\frac{a+f(\alpha) b c}{2 f(\alpha) b}
$$

By bringing the above formula into the profit function (3), (4), it is obtained that:

$$
\pi_{2 e}^{*}=\frac{(a-f(\alpha) b c)^{2}}{16 f(\alpha) b}, \quad \pi_{2 s}^{*}=\frac{(a-f(\alpha) b c)^{2}}{8 f(\alpha) b}
$$

Based on the above models, the profit margin under two different decisions can be obtained as follows:

$$
\Delta \pi=\pi_{1 t}^{*}-\left(\pi_{2 e}^{*}+\pi_{2 s}^{*}\right)=\frac{(a-f(\alpha) b c)^{2}}{16 f(\alpha) b}
$$

From the above models, it can be concluded that under two different decision models, the optimal selling price of fresh products is different, and the total profit of the system under the centralized decision model is larger than the total profit of the system under the decentralized decision model, which indicates that the double marginal effect exists. Therefore, in order to coordinate the supply chain of e-commerce fresh products and maximize the profit of each member, it is necessary to design a reasonable coordination model.

\section{Coordination Model of e-commerce fresh products supply chain based on quantity discount contract}

In order to coordinate the supply chain and be accepted by all members, this paper designs the quantity discount contract $(\lambda, \mathrm{g})$. The supplier is the main party, and the e-commerce platform is the follower. The supplier promises to give the quantity discount of a certain proportion to the e-commerce platform $\lambda(0<\lambda<1)$ on the unit wholesale price according to the total order quantity, and as the condition of encouraging the cooperation, the e-commerce platform must pay the fixed cost $g(g>0)$. Therefore, after the implementation of the quantity discount contract model, the unit wholesale price of fresh products, the profit of e-commerce platform and supplier can be obtained:

$$
\begin{aligned}
& k(\mathrm{~d})=K-\lambda d \\
& \pi_{3 e}=(p-k(d)) d-g=(p-K)(a-f(\alpha) b p)+\lambda(a-f(\alpha) b p)^{\wedge} 2-g \\
& \pi_{3 s}=(k(d)-c) d+g=(K-c)(a-f(\alpha) b p)-\lambda(a-f(\alpha) b p)^{\wedge} 2+g
\end{aligned}
$$

The following propositions can be deduced from the above models:

Proposition 1: Under the quantity discount contract model, for any quantity discount radio and fixed charge, the optimal pricing of fresh products after coordination is $p_{3}{ }^{*}=\frac{a+f(\alpha) b(K-2 a \lambda)}{2 f(\alpha) b(1-\lambda f(\alpha) b)}$.

Prove: By formula (6), taking the first derivative of selling price with the e-commerce fresh produce platform profit is $\frac{\partial \pi_{3 \mathrm{e}}}{\partial p}=a-2 f(\alpha) b+K f(\alpha) b-2 \lambda a b f(\alpha)+2 \lambda f(\alpha)^{2} b^{2} p$, and making the equation to 0. By simplifying the equation, the optimal price is $p_{3}{ }^{*}=\frac{a+f(\alpha) b(K-2 a \lambda)}{2 f(\alpha) b(1-\lambda f(\alpha) b)}$.

Proposition 2: When a quantity discount contract model is adopted, if $(\lambda, g)$ satisfies $K-c=\lambda(a-b c f(\alpha)) \quad$ and $\quad \frac{(1-2 \lambda f(\alpha) b)(a-f(\alpha) b c)^{2}}{8 b f(\alpha)}<g<\frac{(3-4 \lambda f(\alpha) b)(a-f(\alpha) b c)^{2}}{16 b f(\alpha)} \quad$ the e-commerce fresh products supply chain coordination is realized.

Prove: The conclusion that $\lambda$ satisfies $k-c=\lambda(a-b c f(\alpha))$ is derived from $p_{1}^{*}=p_{3}^{*}$. 
If supply chain members are willing to participate in the coordination model, they must make the benefits of coordination greater than those under decentralized decision-making. Then $\pi_{3 s}>\pi_{2 s}, \pi_{3 e}>\pi_{2 e}$,so we can deduce that $g$ meets $g_{1}<g<g_{2}$,so
$\frac{(1-2 \lambda f(\alpha) b)(a-f(\alpha) b c)^{2}}{8 b f(\alpha)}<g<\frac{(3-4 \lambda f(\alpha) b)(a-f(\alpha) b c)^{2}}{16 b f(\alpha)}$.

According to the above proposition, when the price elasticity coefficient of fresh products is invariant, the value of $\lambda$ and the range of value of $g$ will also increase with the increase of freshness of fresh products. This indicates that in the actual sales process, if the freshness of fresh products is fresher, the quantity discount ratio and fixed cost will increase when the quantity discount contract model is implemented. Therefore, in order to achieve win-win situation, supply chain members should maintain freshness of fresh products as much as possible.

Proposition 3. Under a quantity discount contract model, supply chain members allocate increased profit margins based on their bargaining power.

Prove: If $\beta(0<\beta<1)$ is the proportion of fixed cost , the optimal fixed cost is:

$$
g^{*}=(1-\beta) g_{1}+\beta g_{2}
$$

$\mathrm{s}$

By bringing the form (8) into (6), (7), we may obtain:

$$
\pi_{3 e}^{*}=\pi_{2 e}^{*}+(1-\beta) \Delta \pi^{*}, \pi_{3 \mathrm{~s}}^{*}=\pi_{2 s}^{*}+\beta \Delta \pi^{*}
$$

Then $\pi_{3 \mathrm{t}}^{*}=\pi_{3 s}^{*}+\pi_{3 e}^{*}=\pi_{2 s}^{*}+\beta \Delta \pi^{*}+\pi_{2 e}^{*}+(1-\beta) \Delta \pi^{*}=\pi_{2 t}^{*}+\Delta \pi^{*}$

According to proposition 3 , there is still a competitive game after designing the coordination contract model, which needs to decide the size of $\beta(0<\beta<1)$ and the concrete coordination model according to its bargaining power.

\section{Numerical example}

The above is the model analysis, in order to verify its effectiveness, specially set the relevant parameters to verify. The values of the related parameters are assumed to be $a=100, b=0.9, c=10$.

\subsection{Freshness}

Due to fresh products with the characteristics of perishable and prone, this paper assumes that $0.1<\alpha<0.95$. According to daily life experience, when freshness meet $\alpha<0.5$, consumers will not buy fresh products (this article don't consider return). Therefore, this paper only calculates the profit trend chart of freshness satisfying $(0.5 \leq \alpha \leq 0.95)$ under different decisions.

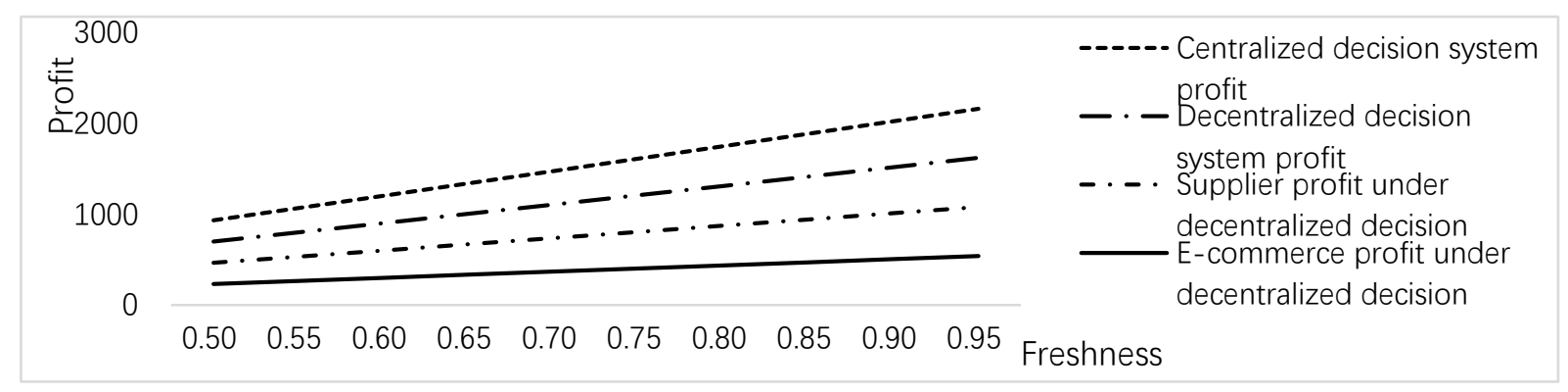

Fig. 1 The influence of freshness on system profit under centralized decision, system, supplier and e-commerce under decentralized decision

As shown in Figure 1, the profits under different decisions will continue to rise as the freshness increases. Through the above calculation, it can be concluded that when the degree of freshness is $\alpha=0.80$, the total profit of the centralized decision system is $\pi_{1 \mathrm{t}}^{*}=1750.35$, and the total profit of the decentralized decision system is $\pi_{2 \mathrm{t}}^{*}=\pi_{2 s}^{*}+\pi_{2 e}^{*}=1312.76$. It shows that the total profit of the system under centralized decision is greater than that of the system under decentralized decision. 
Therefore, it is necessary to design a reasonable coordination model.

\subsection{Model parameter}

In the actual sales process, the fresher the fresh products, the more consumers will be favored. In this paper, we assume that the freshness of fresh products is $\alpha=0.8$, and from proposition 2 we conclude that the value range of $\lambda$ satisfy $0<\lambda<0.37$, when $0<g_{1}, 0<g_{2}$. Therefore, when $\alpha=0.8$ is used, the range of $g$ is shown in Table 1 under the different values of $\lambda$. As can be seen from Table 1, when the discount rate of fresh products increases, the range of fixed costs is declining. Therefore, there is still a game among the members of the supply chain, and it is necessary to strive for more profit margin according to their bargaining power.

Table 1 The range of values of $g$ when $\lambda$ is changed

\begin{tabular}{ccccc|c|c|c|c}
\hline$\lambda$ & 0.00 & 0.05 & 0.10 & 0.15 & 0.20 & 0.25 & 0.0 & 0.35 \\
$g$ & {$[875.17$,} & {$[776.71$,} & {$[678.26$,} & {$[579.79$,} & {$[481.34$,} & {$[382.89$,} & {$[284.43$,} & {$[185.97$,} \\
& $1312.76]$ & $1214.30]$ & $1115.85]$ & $1017.38]$ & $918.93]$ & $820.47]$ & $722.02]$ & $623.56]$ \\
\hline
\end{tabular}

From Table 2, it can be seen that when $\alpha$ and $\lambda$ are determined after coordination, $g^{*}, \pi_{3 \mathrm{~s}}^{*}$ and $\beta$ are in a positive relationship, while $\pi_{3 \mathrm{e}}^{*}$ and $\beta$ are inversely related, and the size of $\beta$ is decided by supply chain members. Therefore, when determining the quantity discount contract model, the supplier should obtain its bargaining power by expanding the quantity discount ratio, while the e-commerce fresh products platform should obtain its bargaining power by reducing the fixed fee.

Table 2 The values of $g^{*}, \pi_{3 \mathrm{~s}}^{*}$ and when $\beta$ is changed

\begin{tabular}{ccccccccccc}
\hline$\beta$ & 0.1 & 0.2 & 0.3 & 0.4 & 0.5 & 0.6 & 0.7 & 0.8 & 0.9 & 0.1 \\
\hline$g^{*}$ & 680.0 & 717.0 & 753.9 & 790.9 & 827.8 & 864.8 & 901.7 & 938.7 & 975.6 & 1012.6 \\
$\pi_{3 \mathrm{~s}}^{*}$ & 778.6 & 815.5 & 852.5 & 889.4 & 926.4 & 963.3 & 1000.3 & 1037.2 & 1074.2 & 1111.1 \\
$\pi_{3 \mathrm{e}}^{*}$ & 971.2 & 934.3 & 897.4 & 860.4 & 823.5 & 786.5 & 749.6 & 712.6 & 675.7 & 638.7 \\
\hline
\end{tabular}

\section{Conclusion}

In this paper, according to the unique product characteristics of fresh products, the influence factors of freshness on demand are added. Firstly, the decision model is established, and the profit margin of supply chain under two different decision models is deduced through the analysis of the model. Then the supply chain is coordinated by constructing a coordinated decision model. Through numerical simulation, it is found that under different freshness, the total profit of e-commerce fresh products supply chain under centralized decision model is larger than that under decentralized decision model, and the influence of model parameters on optimal profit is quantitatively analyzed. Finally the results show that the coordination is achieved by coordination model, but the supply chain members have to rely on their bargaining power to earn more profit margin.

However, the freshness of fresh products will change with time, and the transportation cost, storage cost and return cost caused by time factors are not considered in this paper. The factors such as transportation lead to the decline of freshness, which is the focus of follow-up research.

\section{References}

[1] Sun Guohua, Ge Yan.Strategy for Optimizing and Coordinating Dual-channel Fresh Product Supply Chain [J].Logistics Technology, 2018(4):112-131.

[2] DAN Bin, XU Guang-ye.A Compensation Strategy for Coordinating Dual-channel Supply Chains in E-commerce[J].Journal of Industrial Engineering,2012(1):125-130.

[3] Amini M, Li HT. The Impact of Dual-market on Supply Chain Configuration for New Products 
[J].International Journal of Production Research, 2015(18):5669-5684.

[4] Dan Bin, Fu Hongyong.Coordination of Agri-food Supply Chain with Weather-related Yield under Risk-averse Producer [J].Journal of Systems Engineering, 2014(3):362-370.

[5] Tang Run.Supply Chain Profit Sharing Contract of Fresh Agricultural Products under the Influence of Fresh-keeping Temperature [J].Jiangsu Agricultural Sciences, 2017(1):293-298.

[6] Chen Yijuan.Dynamic Pricing Model of Fresh Food Considering Freshness [J].Statistics \& Decision, 2016(8):47-50. 